Bull. Austral. Math. Soc.

$30 \mathrm{G} 30,46 \mathrm{E} 10$

VoL. 42 (1990) [369-382]

\title{
ANALYTICITY AND QUASI-BANACH VALUED FUNCTIONS
}

\author{
Antonio Bernal and Joan Cerdà
}

We compare the definitions of analyticity of vector-valued functions and their connections with the topological tensor products of non-locally convex spaces.

\section{INTRODUCTION}

In this paper we consider quasi-Banach valued functions that are defined on an open subset of the complex plane.

For such a function, there are several definitions for the concept of analyticity. Complex differentiability allows as analytic some functions that are too pathological (see $[1,4,12])$. Other definitions have been considered by several authors in $[1,4$, $5,7,8,11,12]$, and the proof of the equivalence of these definitions is more or less implicit in these papers.

Our purpose is to give a unified presentation of the basic theory of analytic functions with values in quasi-Banach spaces and to prove the above mentioned equivalences in full detail.

Section 2 contains the preliminary definitions, notation and results on analyticity. In Section 3 we prove that the uniform limits of finite rank analytic functions have power series expansions. This fact has been shown by Kalton in [8] but here we give another proof that gives a more elementary appearance to the theorem.

In Sections 4 and 5 we complete the chain of equivalences for the different definitions of analy ticity and we observe that the concept of ultra-uniform convergence on compact sets introduced by Etter in [4], which is known to be strictly stronger than the uniform convergence on compact sets, is equivalent to it when we consider analytic functions.

The proof of the result of Section 5 uses some facts about the theory of non locally convex tensor products as developed in the papers $[2,5,6,7,13,14,15,16]$.

Throughout the paper $G$ represents a domain of the complex plane and $(X,\|\cdot\|)$ a complex quasi-Banach space. We refer to [2] and [10] for precise definitions and basic properties of quasi-Banach spaces. Throughout the paper we shall assume all the quasinorms to be $p$-norms for a certain $p, 0<p \leqslant 1$. This can be done since a theorem of

Received 16 November 1989

This research was partially supported by DGICYT, Grant PS87-0027.

Copyright Clearance Centre, Inc. Serial-fee code: 0004-9729/90 \$A2.00+0.00. 
Aoki and Rolewicz asserts that every quasi-norm on a linear space is equivalent to a $p$-norm with suitable $p, 0<p \leqslant 1$.

We also make free use of the notations on the non locally convex tensor products $X \widehat{\otimes}_{p} Y$ and $X \widehat{\otimes}_{\epsilon} Y, Y$ being a locally convex space with generating system of seminorms $\left\{\|\cdot\|_{\alpha}\right\}_{\alpha}$ and basis of zero-neighbourhoods $\left\{U_{\beta}\right\}_{\beta}$.

Recall that $X \widehat{\otimes}_{p} Y$ stands for the completion of the tensor product $X \otimes Y$ with respect to the quasi-seminorms

$$
\|\cdot\| \otimes_{p}\|\cdot\|_{\alpha}(Z)=\inf \left(\sum\left\|x_{n}\right\|^{p}\left\|y_{n}\right\|_{\alpha}^{p}\right)^{1 / p}, .
$$

where the infimum is taken over all finite representations

$$
Z=\sum x_{n} \otimes y_{n}
$$

Also, $X \widehat{\otimes}_{\varepsilon} Y$ is the completion of $X \otimes Y$ with respect to the quasi-seminorms

$$
q_{\beta}(Z)=\sup \left\|\left(I_{X} \otimes \omega\right)(Z)\right\|,
$$

the supremum being taken over all the continuous linear functionals $\omega \in Y^{\prime}$ which are in the polar of $U_{\beta}$.

\section{DEFINITIONS OF ANALYTICITY}

In this section we give several definitions for the concept of analyticity and we prove some relations between them.

Let $f_{n}$ be a sequence of $X$-valued functions defined on $G$. In [4], $f_{n}$ is said to be ultra-uniformly convergent to $f: G \rightarrow X$ over a set $A \subset G$ if to each zeroneighbourhood $W$ on $X$ there corresponds an index $n_{0}$ so that

$$
\operatorname{co}\left[\left(f_{n}-f\right)(A)\right] \subset W
$$

whenever $n \geqslant n_{0}$. Here "co" denotes the convex envelope of the set.

In [4], a function $f: G \rightarrow X$ is said to be of class $A$ if there is a sequence of finite rank analytic functions which is ultra-uniformly convergent to $f$ over each compact subset of $G$.

From this definition an integration theory is developed in [4] such that the Cauchy integral formula holds for functions of class $A$. However, this integration theory gives no mean value inequality and, if $\gamma$ is a rectifiable curve in $G$, no estimate for the quasi-norm of $\int_{\gamma} f(z) d z$ can be obtained from the supremum of $\|f(z)\|$ over $\gamma$.

The fundamental property of functions of class $A$ is the following factorisation theorem of [4]. 
THEOREM 2.1. A function $f: G \rightarrow X$ is of class $A$ if and only if for each $z \in G$ there is a disk $D(z) \subset G$ centred at $z$, a Banach space $L_{z}$, a linear operator $T_{z}: L_{z} \rightarrow X$ and a Banach-valued analytic function

$\begin{array}{cc} & f_{z}: D(z) \rightarrow L_{z} \\ \text { such that } & \left.f\right|_{D(z)}=T_{z} \circ f_{z}\end{array}$

In [4] it is also proved that, if $z_{0} \in G$, any function of class $A$ in $G$ has a power series expansion which is ultra-uniformly convergent on any closed disk $\bar{D}\left(z_{0}\right) \subset G$. From this fact it is natural to make the following definition:

A function $f: G \rightarrow X$ will be said to be analytic when for each $z_{0}$ there is a disk $D\left(z_{0}\right) \subset G$ centred at $z_{0}$ and a power series expansion

$$
f(z)=\sum_{n=0}^{\infty}\left(z-z_{0}\right)^{n} a_{n}, \quad a_{n} \in X
$$

which is pointwise convergent on $D\left(z_{0}\right)$.

Thus, any function of class $A$ is analytic.

Since $X$ is a quasi-Banach space, the usual arguments work to prove that the above series is uniformly convergent on compact subsets of $D\left(z_{0}\right)$. We will see that the series (1) is ultra-uniformly convergent on compact sets and that the class $A$ and the class of all analytic functions are the same.

Another definition has been given by Gramsch in [5]. For the moment we will call locally holomorphic a function $f: G \rightarrow X$ if locally belongs to $X \widehat{\otimes}_{p} H^{\infty}$. More explicitly: if for each $z_{0} \in G$ there is an open set $U\left(z_{0}\right) \subset G$ containing $z_{0}$, vectors $x_{k} \in X$ and bounded scalar-valued analytic functions $f_{k}: U\left(z_{0}\right) \rightarrow C$ such that

$$
\sum_{k=1}^{\infty}\left\|x_{k}\right\|^{p}\left\|f_{k}\right\|_{\infty}^{p}<+\infty
$$

and

$$
f(z)=\sum_{k=1}^{\infty} f_{k}(z) x_{k}
$$

in $U\left(z_{0}\right)$. We remark that (2) implies the convergence of (3). At this moment we are not saying that the completed tensor products $X \widehat{\otimes}_{p} H^{\infty}\left(U\left(z_{0}\right)\right)$ are function spaces.

We observe that analytic functions, locally holomorphic functions and functions of class $A$ are the same: 
Any function of class $A$ is analytic as we have seen. But analytic functions with values in very general spaces have been studied by Turpin in [12] and in the quasinormed case the factorisation theorem is proved for such functions (the disk $D(z)$ of Theorem 2.1 can be replaced by any open set $U$ so that $\bar{U}$ is a compact subset of $G$ ) [12, Theorem 9.3.2]. Thus, by Theorem 2.1, any analytic function is of class $A$. Moreover, taking the power series expansions, it follows that analytic functions are locally holomorphic and in [5] the reverse implication has been proved.

Proposition 2.1. For a function $f: G \rightarrow X$ the following properties are equivalent:

(i) $f$ is of class $A$.

(ii) $f$ is analytic.

(iii) $f$ is locally holomorphic.

The factorisation property proved by Turpin for analytic functions allows us to prove local properties of analytic functions from the Banach-valued case. For example, it follows that analytic functions are $\mathcal{C}^{\infty}$ and the relations

$$
a_{k}=\frac{1}{k !} f^{(k)}\left(z_{0}\right)
$$

hold for the coefficients of the power series (1), and the power series of $f$ about $z_{0}$ converges on any disk $D\left(z_{0}\right) \subset G$ centred at $z_{0}$.

We remark that in the definition of local holomorphic functions we can use any $p(0<p \leqslant 1)$ so that $X$ is $p$-Banach, because it follows from the above proposition that the class of all locally holomorphic functions is independent of $p$.

We introduce two further definitions:

We shall say that $f$ is globally holomorphic if there is a sequence $f_{k}: G \rightarrow C$ of scalar-valued analytic functions and a sequence of vectors $x_{k} \in X$ such that, if $K \subset G$ is compact, then

and

$$
\begin{gathered}
\sum_{k=1}^{\infty}\left\|x_{k}\right\|^{p}\left\|f_{k}\right\|_{K}^{p}<+\infty \\
f(z)=\sum_{k=1}^{\infty} f_{k}(z) s_{k}
\end{gathered}
$$

over $G$. Here $\left\|f_{k}\right\|_{K}$ stands for the maximum of $\left|f_{k}(z)\right|$ over $K$. This definition has been considered in [7].

We shall call $f$ holomorphic if there is a sequence of analytic functions with finite rank $f_{n}: G \rightarrow X$ uniformly convergent on compact sets of $G$ to $f$. This definition is due to Peetre in [11]. 
Obviously, any globally holomorphic function is analytic and any analytic function is holomorphic. We shall prove the converse of these two properties.

\section{ANALYTICITY OF HOLOMORPHIC FUNCTIONS}

In [8, Theorem 6.3] Kalton considers the space $A_{0}(X)$ of all continuous functions $f: \bar{\Delta} \rightarrow X, \Delta$ being the unit disk, which are analytic on $\Delta$, and shows that $A_{0}(X)$ is complete with respect to the quasi-norm

$$
\|f\|_{\infty}=\max _{|z| \leqslant 1}\|f(z)\| .
$$

Thus the uniform limit of a sequence of analytic functions is analytic and any holomorphic function is analytic.

The proof of the completeness of $A_{0}(X)$ given in [8] is connected with the atomic theory of $H^{p}$. It uses a result by Coifman and Rochberg [3] on Bergman spaces.

We shall give another proof which uses a less involved concept of complex analysis: the maximum principle. Since the maximum principle is not valid in general quasiBanach spaces (see $[\mathbf{1}]$ and $[9]$ ) we shall use the generalisation of the maximum principle given in [8].

The proof of this generalisation also uses the above mentioned techniques and so our proof is only apparently more elementary than that of [8]. Nevertheless, the class of all quasi-Banach spaces for which the maximum principle is valid contains important examples such as $L^{p}$ and $H^{p}$. These spaces have been called "locally analytically pseudoconvex" by Peetre in [11], "locally holomorphic" by Aleksandrov in $[1]$ and " $A$ convex" by Kalton in [9]. For an $A$-convex space our proof is actually more elementary than that of [0].

Recall that $X$ is $A$-convex if it has an equivalent pluri-subharmonic quasi-norm. This property is characterised by the existence of a constant $C$ such that, if $f$ is an $X$-valued analytic function on $\Delta$ which is continuous up to the boundary, then

$$
\|f(0)\| \leqslant C \sup _{|z|=1}\|f(z)\| .
$$

If $X$ is $A$-convex, we take an equivalent $p$-norm $|\cdot|$ and $(X,|\cdot|)$ is a $p$-Banach space satisfying (4). According to [9, Theorem 3.7], we can suppose that the $p$-norm is pluri-subharmonic and in (4) we have $C=1$.

Lemma 3.1. Let $g: \bar{\Delta} \rightarrow X$ be continuous on $\bar{\Delta}$ and holomorphic on $\Delta$. Let $r$ satisfy $0<r<1$. Then there is a constant $C=C(r, X)$ with

$$
\|f(0)\| \leqslant C \sup _{r \leqslant|z| \leqslant 1}\|g(z)\| .
$$


Proof: If $g$ is analytic, this is [9, Theorem 5.2]. If not, we just make a limiting process approximating $f$ on $\bar{\Delta}$ by finite rank functions which are analytic on a neighbourhood of $\bar{\Delta}$.

We remark that the last case of the proof will never happen because we shall prove that any holomorphic function is analytic.

Now take a holomorphic function $f: G \rightarrow X$ and a sequence $f_{n}: G \rightarrow X$ of analytic functions with finite rank uniformly convergent to $f$ over the compact sets of $G$. Fix $z_{0} \in G$ and $r_{0}>0$ so that $\bar{D}\left(z_{0}, r_{0}\right) \subset G$. We have the power series for the functions $f_{n}$

$$
f_{n}(z)=\sum_{k=0}^{\infty}\left(z-z_{0}\right)^{k} a_{k}^{(n)}
$$

LEMMA 3.2. For each integer $k \geqslant 0$ the sequence $\left\{a_{k}^{(n)}\right\}_{n}$ is convergent.

Proof: The result is obvious for $k=0$. Suppose that

$$
a_{k}^{(n)} \rightarrow a_{k}
$$

as $n \rightarrow \infty$ if $0 \leqslant k<m$. If $z \in \bar{D}\left(z_{0}, r_{0}\right) \backslash\left\{z_{0}\right\}$ we write

$$
\frac{f_{n}(z)}{\left(z-z_{0}\right)^{m}}=\frac{a_{0}^{(n)}}{\left(z-z_{0}\right)^{m}}+\frac{a_{1}^{(n)}}{\left(z-z_{0}\right)^{m-1}}+\cdots+\frac{a_{m-1}^{(n)}}{z-z_{0}}+a_{m}^{(n)}+\left(z-z_{0}\right) a_{m+1}^{(n)}+\cdots
$$

We know that the power series $\sum_{\nu=0}^{\infty}\left(z-z_{0}\right)^{\nu} a_{m+\nu}^{(n)}$ are pointwise convergent on a neighbourhood of $\bar{D}\left(z_{0}, r_{0}\right)$ and with the usual arguments it follows that they are uniformly convergent on $\bar{D}\left(z_{0}, r_{0}\right)$. Let us call $h_{n m}$ the functions defined on $\bar{D}\left(z_{0}, r_{0}\right)$ by the above power series. They are holomorphic on $D\left(z_{0}, r_{0}\right)$. For $z \in \bar{D}\left(z_{0}, r_{0}\right) \backslash\left\{z_{0}\right\}$ the following identity holds:

$$
h_{n m}(z)=\frac{f_{n}(z)}{\left(z-z_{0}\right)^{m}}-\frac{a_{0}^{(n)}}{\left(z-z_{0}\right)^{m}}-\cdots-\frac{a_{m-1}^{(n)}}{z-z_{0}} .
$$

By the inductive hypothesis $h_{n m}(z) \rightarrow g_{m}(z)$ pointwise on $\bar{D}\left(z_{0}, r_{0}\right) \backslash\left\{z_{0}\right\}$, where $g_{m}$ is the function defined on $\bar{D}\left(z_{0}, r_{0}\right) \backslash\left\{z_{0}\right\}$ by

$$
g_{m}(z)=\frac{f(z)}{\left(z-z_{0}\right)^{m}}-\frac{a_{0}}{\left(z-z_{0}\right)^{m}}-\cdots-\frac{a_{m-1}}{z-z_{0}} .
$$

Moreover, if $0<r<r_{0}$, the convergence is uniform on the ring of centre $z_{0}$ and radii $r$ and $r_{0}$ :

$$
\left\|h_{n m}-g_{m}(z)\right\|^{p} \leqslant \frac{1}{r^{m p}}\left\|f_{n}(z)-f(z)\right\|^{p}+\cdots+\frac{1}{r^{p}}\left\|a_{m-1}^{(n)}-a_{m-1}\right\|^{p} .
$$


In particular, the sequence $\left\{h_{n m}\right\}_{n}$ is uniformly Cauchy on the ring of radii $r$ and $r_{0}$. From this and from Lemma 3.1 it follows that $a_{m}^{(n)}=h_{n m}\left(z_{0}\right)$ is convergent in $X$ as $n \rightarrow \infty$. This proves the lemma.

We now have a candidate for power series of $f$ about $z_{0}$, formally

$$
f(z)=\sum_{\nu=0}^{\infty}\left(z-z_{0}\right)^{\nu} a_{\nu}
$$

where, according to the above notation, $a_{\nu}=\lim _{n \rightarrow \infty} a_{\nu}^{(n)}$. Before proving that the series (5) converges to $f(z)$ on a neighbourhood of $z_{0}$, we state a technical lemma.

Lemma 3.3. The functions $h_{m}$ defined on $\bar{D}\left(z_{0}, r_{0}\right)$ by

$$
h_{m}(z)= \begin{cases}g_{m}(z) & \text { if } z \neq z_{0} \\ a_{m} & \text { otherwise }\end{cases}
$$

are holomorphic.

Proof: Since $h_{n m}$ are analytic on $D\left(z_{0}, r_{0}\right)$, it is enough to prove that $h_{n m} \rightarrow h_{m}$ uniformly on $\bar{D}\left(z_{0}, r_{0}\right)$.

As we have seen in the proof of Lemma 3.2, $h_{n m} \rightarrow h_{m}$ uniformly on the ring of centre $z_{0}$ and radii $r_{0}$ and $r_{0} / 2$.

From Lemma 3.1 plus a conformal mapping argument it follows that, if $h: \bar{D}\left(z_{0}, r_{0}\right) \rightarrow X$ is continuous and holomorphic on $D\left(z_{0}, r_{0}\right)$, there is a constant $C$ and a radius $r, 0<r<r_{0}$, so that

$$
\sup _{\left|z-z_{0}\right| \leqslant r_{0} / 2}\|h(z)\| \leqslant C \cdot \sup _{r \leqslant\left|z-z_{0}\right| \leqslant r_{0}}\|h(z)\| .
$$

From this observation it follows that the sequence $\left\{h_{n m}\right\}_{n}$ is uniformly Cauchy on the disk $\bar{D}\left(z_{0}, r_{0} / 2\right)$, but $h_{n m} \rightarrow h_{m}$ pointwise on $D\left(z_{0}, r_{0}\right)$.

We can now state the main result.

THEOREM 3.1. The series (5) is convergent to $f(z)$ for all $z$ in a certain neighbourhood of $z_{0}$. Thus (5) is also convergent on any disk contained in $G$ centred at $z_{0}$ and any holomorphic function is analytic.

Proof: If $r_{0} / 2 \leqslant\left|z-z_{0}\right| \leqslant r_{0}$, it follows that

$$
h_{n m}(z)-h_{m}(z)=\frac{f_{n}(z)-f(z)}{\left(z-z_{0}\right)^{m}}-\frac{a_{0}^{(n)}-a_{0}}{\left(z-z_{0}\right)^{m}}-\frac{a_{1}^{(n)}-a_{1}}{\left(z-z_{0}\right)^{m-1}}-\cdots-\frac{a_{m-1}^{(n)}-a_{m-1}}{z-z_{0}} .
$$


From this we have

$$
\begin{aligned}
\left\|\left(\frac{r_{0}}{2}\right)^{m}\left[h_{n m}(z)-h_{m}(z)\right]\right\|^{p} \leqslant & \left\|f_{n}(z)-f(z)\right\|^{p}+\left\|a_{0}^{(n)}-a_{0}\right\|^{p} \\
& +\left\|\frac{r_{0}}{2}\left(a_{1}^{(n)}-a_{1}\right)\right\|^{p}+\cdots \\
& +\left\|\left(\frac{r_{0}}{2}\right)^{m-1}\left(a_{m-1}^{(n)}-a_{m-1}\right)\right\|^{p}
\end{aligned}
$$

and, applying Lemma 3.1,

$$
\begin{aligned}
& \left\|\left(\frac{r_{0}}{2}\right)^{m}\left(a_{m}^{(n)}-a^{m}\right)\right\|^{p} \\
& \quad \leqslant C\left(\left\|f_{n}-f\right\|^{p}+\left\|a_{0}^{(n)}-a_{0}\right\|^{p}+\cdots+\left\|\left(\frac{r_{0}}{2}\right)^{m-1}\left(a_{m-1}^{(n)}-a_{m-1}\right)\right\|^{p}\right)
\end{aligned}
$$

where $\left\|f_{n}-f\right\|$ stands for the supremum of $\left\|f_{n}(z)-f(z)\right\|$ over the disk $\bar{D}\left(z_{0}, r_{0}\right)$.

From the last inequality it follows by an inductive argument:

$$
\begin{aligned}
\left\|f_{n}-f\right\|^{p} & +\left\|a_{0}^{(n)}-a_{0}\right\|^{p}+\cdots+\left\|\left(\frac{r_{0}}{2}\right)^{m-1}\left(a_{m-1}^{(n)}-a_{m-1}\right)\right\|^{p} \\
& \leq(1+C)^{m-1}\left(\left\|f_{n}-f\right\|^{p}+\left\|a_{0}^{(n)}-a_{0}\right\|^{p}\right)
\end{aligned}
$$

and we arrive at

$$
\left\|a_{m}^{(n)}-a_{m}\right\| \leqslant C^{m}\left\|f_{n}-f\right\|,
$$

where the constant $C$ is independent of $m$ and $n$.

The argument up to this point could have been used to obtain estimates for the Taylor coefficients of analytic functions, but the estimates in $[8$, Theorem 6.1$]$ are more precise.

Now pick $z_{1}$ such that $\left|z_{1}-z_{0}\right|<(2 C)^{-1}$. It follows that

and so

$$
\sum_{m=0}^{\infty} C^{m p}\left\|f_{n}-f\right\|^{p}\left|z_{1}-z_{0}\right|^{p m}<+\infty
$$

$$
\sum_{m=0}^{\infty}\left\|\left(z_{1}-z_{0}\right)^{m}\left(a_{m}^{(n)}-a_{m}\right)\right\|^{p} \rightarrow 0
$$

as $n \rightarrow \infty$. We have proved that the series $\sum_{m=0}^{\infty}\left(z_{1}-z_{0}\right)^{m} a_{m}$ has non null radius of convergence. Moreover, we know that

and

$$
\begin{gathered}
\left\|f_{n}\left(z_{1}\right)-f\left(z_{1}\right)\right\| \rightarrow 0 \\
\left\|f_{n}\left(z_{1}\right)-\sum_{m=0}^{\infty}\left(z_{1}-z_{0}\right)^{m} a_{m}\right\| \rightarrow 0,
\end{gathered}
$$

as $n \rightarrow \infty$. This completes the proof of the theorem. 


\section{Convergence and Ultra-Convergence}

In [5] an example is given of an $l^{P}$-valued function, with $p=1 / 3$, that can be uniformly approximated by continuous functions with finite rank but fails to be integrable. Since ultra-uniform convergence preserves integrability of quasi-Banach valued functions as was proved in [4], it follows that the ultra-uniform convergence is a concept strictly stronger than uniform convergence. We prove next that, if we consider only analytic functions, both concepts are essentially equivalent.

If $\left\{K_{n}\right\}_{n}$ is an exhaustive sequence of compact sets of $G$ and $f: G \rightarrow X$ is analy tic, Etter [4] proves that the supremum

$$
u_{n}(f)=\sup \left\{|\xi|: \xi \in \Gamma\left(f\left(K_{n}\right)\right)\right\}
$$

is finite, where $\Gamma(A)$ stands for the set of all finite combinations $\sum \lambda_{i} a_{i}$, with $a_{i} \in A$, $\lambda_{i} \in C$ and $\sum\left|\lambda_{i}\right| \leqslant 1$. For a sequence of functions $\left\{f_{\nu}\right\}_{\nu}$ in $\mathcal{H}(G, X)$ the statements " $u_{n}\left(f_{\nu}\right) \rightarrow 0$ as $\nu \rightarrow \infty$ for each $n$ ", and " $\left\{f_{\nu}\right\}_{\nu}$ is ultra-uniformly convergent to zero over each compact subset of $G$ " are equivalent. The functionals $u_{n}$ are $p$-norms that generate on $\mathcal{H}(G, X)$ a locally $p$-convex metrisable topology whose convergence is the ultra-uniform convergence on compact subsets of $G$.

Proposition 4.1. The space $\mathcal{H}(G, X)$ is complete with sespect to the quasinorms $u_{n}$.

Proof: Let $\left\{f_{n}\right\}_{n}$ be a Cauchy sequence. Consider a subsequence $f_{n_{m}}$ so that

$$
\Gamma\left[\left(f_{n_{m+1}}-f_{n_{m}}\right)\left(K_{m}\right)\right] \subset B_{X}\left(0,2^{-m / p}\right) .
$$

If $m \geqslant 2$, it follows that

$$
f_{n_{m}}=f_{n_{1}}+\sum_{k=1}^{m-1}\left(f_{n_{k+1}}-f_{n_{k}}\right) .
$$

The series $\sum_{k=1}^{\infty}\left(f_{n_{k+1}}-f_{n_{k}}\right)$ is uniformly convergent over compact subsets of $G$ to a function $\psi: G \rightarrow X$. If $h$ is a positive integer and $\varepsilon>0$, pick an integer $N$ so that

$$
\sum_{k=N}^{\infty} 2^{-k}<\varepsilon^{p} \text { and } K_{h} \subset K_{N}
$$

If $m \geqslant N$ and $z \in K_{h}$, it follows that, denoting $f=f_{n_{1}}+\psi$,

$$
f(z)-f_{n_{m}}(z)=\sum_{k=m}^{\infty}\left[f_{n_{k+1}}(z)-f_{n_{k}}(z)\right]
$$


Then, for any $y \in \Gamma\left[\left(f-f_{n_{m}}\right)\left(K_{h}\right)\right]$, it will follow that

$$
y=\sum_{k=m}^{\infty} \sum_{i=1}^{r} \lambda_{i}\left[f_{n_{k+1}}\left(z_{i}\right)-f_{n_{k}}\left(z_{i}\right)\right],
$$

with $z_{i} \in K_{h}, \lambda_{i} \in C$ and $\sum\left|\lambda_{i}\right| \leqslant 1$.

From this it follows that $\Gamma\left(f-f_{n_{m}}\right)\left(K_{h}\right) \subset B_{X}(0, \varepsilon)$. We have proved that $f_{n_{m}} \rightarrow$ $f$ and $m \rightarrow \infty$, ultra-uniformly over compact subsets of $G$.

THEOREM 4.1. Let $f_{n}: G \rightarrow X$ be analytic. If $\left\{f_{n}\right\}_{n}$ is uniformly convergent on compact sets of $G$, then $\left\{f_{n}\right\}_{n}$ is also ultra-uniformly convergent on compact sets to the same limit.

ProOF: According to the last proposition, the space $\mathcal{H}(G, X)$ endowed with the $p$-norms $\left\{u_{k}\right\}_{k}$ is a complete metrisable linear space. So it is with respect to the p-norms

$$
p_{n}(f)=\sup _{z \in K_{n}}\|f(z)\|
$$

according to Proposition 4.1. Since $p_{n} \leqslant u_{n}$, we can use the open mapping theorem.

\section{GLOBAL HOLOMORPHY OF ANALYTIC FUNCTIONS}

Let $G,\left\{p_{n}\right\}_{n},\left\{u_{n}\right\}_{n}$ and $\left\{K_{n}\right\}_{n}$ be as in the preceding section. We can identify $X \otimes \mathcal{H}(G)$ with the space of all finite rank $X$-valued analytic functions on $X$. By Theorem 3.1, $\mathcal{H}(G, X)$ is the completion of $X \otimes \mathcal{H}(G)$ with respect to the p-norms $\left\{p_{n}\right\}_{n}$.

Let $U_{n}$ be the set of all scalar valued $\phi \in \mathcal{H}(G)$ such that $\sup _{z \in K_{n}}|\phi(z)| \leqslant 1$, a basic zero-neighbourhood in $\mathcal{H}(G)$. Recall that the functionals $q_{n}$ defined for $g \in X \otimes \mathcal{H}(G)$ by

$$
q_{n}(g)=\sup _{\omega \in U_{n}^{0}}\left\|\left(I_{X} \otimes \omega\right)(g)\right\|
$$

define the inductive topology on $X \otimes \mathcal{H}(G)$. Also $\delta_{z} \in U_{n}^{0}$ if $z \in K_{n}$. From this it follows that $p_{n}(g) \leqslant q_{n}(g)$ for any $g \in X \otimes \mathcal{H}(G)$.

In the locally convex case the equality of $p_{n}$ and $q_{n}$ can be proved. Suppose that $X$ is a Banach space and denote by $\Delta_{n}$ the set of all $\delta_{z}$ with $z \in K_{n}$. Then $U_{n}=\Delta_{n}^{0}$ and

$$
\overline{\Gamma \Delta_{n}}=U_{n}^{0},
$$

and the closure can be taken in the weak topology of the topological dual $\mathcal{H}^{\prime}(G)$. 
If $\lambda_{i}, 1 \leqslant i \leqslant r$, are scalars so that $\sum\left|\lambda_{i}\right| \leqslant 1, z_{i} \in K_{n}, g=\sum x_{i} \otimes \phi_{i} \in X \otimes \mathcal{H}(G)$ and $\omega=\lambda_{1} \delta_{z_{1}}+\cdots+\lambda_{r} \delta_{z_{r}}$, then

$$
\begin{aligned}
\left\|\sum\left\langle\omega, \phi_{i}\right) x_{i}\right\| & \leqslant \sum\left|\lambda_{j}\right|\left\|\sum \phi_{i}\left(z_{j}\right) x_{i}\right\| \\
& \leqslant p_{n}(g) .
\end{aligned}
$$

If $\omega \in U_{n}^{0}$, we approximate by $\omega_{\alpha} \in \Gamma\left(\Delta_{n}\right)$ and the same result follows. Thus $q_{n}(g) \leqslant p_{n}(g)$.

But in our case we do not have inequality (6). It is true however that the topologies generated in $X \otimes \mathcal{H}(G)$ by $\left\{p_{n}\right\}_{n}$ and $\left\{q_{n}\right\}_{n}$ are the same:

Proposition 5.1. The continuous extension

of the identity

$$
\Phi: X \widehat{\otimes}_{\varepsilon} \mathcal{H}(G) \rightarrow \mathcal{H}(G, X)
$$

is a topological isomorphism.

Proof: This result has been proved in [7] using some considerations on the tensor product $X \widehat{\otimes}_{e} \mathcal{C}(G)$. We give a direct proof.

The inequality $p_{n} \leqslant q_{n}$ extends to the completion

$$
p_{n}(\Phi(u)) \leqslant \check{q}_{n}(u)
$$

for each $u \in X \widehat{\otimes}_{\varepsilon} \mathcal{H}(G)$.

We prove that $\Phi$ is one to one. Let $u \in X \widehat{\otimes}_{\varepsilon} \mathcal{H}(G)$ be so that $\Phi(u)=0$. We show that $\dot{q}_{n}(u)=0$ for each $n$. The quasi-norms $\ddot{q}_{n}$ have the representation

$$
\bar{q}_{n}(u)=\sup _{\omega \in U_{n}^{0}}\left\|I_{X} \otimes \omega(u)\right\|,
$$

where $I_{X} \dot{\otimes} \omega$ denotes the extension to $X \widehat{\otimes}_{\mathcal{C}} \mathcal{H}(G)$ of the continuous linear mapping

$$
I_{X} \otimes \omega: X \otimes_{c} \mathcal{H}(G) \rightarrow X .
$$

See [7] or [2, Proposition 3.1].

Thus we have to prove that

$$
I_{X} \otimes \omega(u)=0
$$

if $\omega \in U_{n}^{0}=\overline{\Gamma \Delta_{n}}$. Consider first $\omega \in \Gamma \Delta_{n}, \varepsilon>0, \omega=\sum_{i=1}^{\Gamma} \lambda_{i} \delta_{z_{i}}$, with $\lambda_{i} \in C \backslash\{0\}$ so that $\sum\left|\lambda_{i}\right| \leqslant 1$ and $z_{i} \in K_{n}$. Pick $u^{\prime} \in X \otimes \mathcal{H}(G)$ with $\check{q}_{n}\left(u-u^{\prime}\right)<\varepsilon\left(1+\sum\left|\lambda_{i}\right|^{p}\right)^{-1 / p}$. Then

$$
p_{n}\left(u^{\prime}\right)=p_{n}\left(\Phi\left(u-u^{\prime}\right)\right) \leqslant \check{q}_{n}\left(u-u^{\prime}\right)<\varepsilon\left(1+\sum\left|\lambda_{i}\right|^{p}\right)^{-1 / p}
$$

and

$$
\left\|I_{X} \dot{\otimes} \omega(u)\right\|^{p} \leqslant \check{q}_{n}\left(u-u^{\prime}\right)^{p}+\left\|I_{X} \otimes \omega\left(u^{\prime}\right)\right\|^{p} .
$$


Now suppose that $u^{\prime}=\sum x_{j} \otimes \phi_{j} \in X \otimes \mathcal{H}(G)$. Then

$$
\begin{aligned}
\left\|\left(I_{X} \otimes \omega\right)\left(u^{\prime}\right)\right\|^{p} & =\left\|\sum_{i} \lambda_{i} \sum_{j} \phi_{j}\left(z_{i}\right) x_{j}\right\|^{p} \\
& \leqslant \sum_{i}\left|\lambda_{i}\right|^{p}\left\|\sum_{j} \phi_{j}\left(z_{i}\right) x_{j}\right\|^{p} \\
& \leqslant p_{n}\left(u^{\prime}\right)^{p} \sum_{i}\left|\lambda_{i}\right|^{p}<\varepsilon^{p} .
\end{aligned}
$$

Thus $\left\|I_{X} \dot{\otimes} \omega(u)\right\|=0$ if $\omega \in \Gamma \Delta_{n}$.

If $\omega \in U_{n}^{0}$, we take again $u^{\prime \prime} \in X \otimes \mathcal{H}(G)$ with

$$
\tilde{q}_{n}\left(u-u^{\prime \prime}\right)^{p}<\varepsilon
$$

With $u^{\prime \prime}$ fixed, we take $\omega^{\prime} \in \Gamma \Delta_{n}$ with

$$
\left\|I_{X} \otimes\left(\omega-\omega^{\prime}\right)\left(u^{\prime \prime}\right)\right\|^{p}<\varepsilon
$$

Now we have

$$
\begin{aligned}
\left\|I_{X} \dot{\otimes} \omega(u)\right\|^{p} & \leqslant\left\|I_{X} \dot{\otimes} \omega\left(u-u^{\prime \prime}\right)\right\|^{p}+\left\|I_{X} \dot{\otimes}\left(\omega-\omega^{\prime}\right)\left(u^{\prime \prime}\right)\right\|^{p} \\
& +\left\|I_{X} \dot{\otimes} \omega^{\prime}\left(u-u^{\prime \prime}\right)\right\|^{p}+\left\|I_{X} \dot{\otimes} \omega^{\prime}(u)\right\|^{p} .
\end{aligned}
$$

As we have seen before, the last term of the sum vanishes and we have $\left(\omega, \omega^{\prime} \in U_{n}^{0}\right)$ :

$$
\left\|I_{X} \check{\otimes} \omega(u)\right\|^{p}<3 \varepsilon
$$

and the one-to-one character of $\Phi$ is proved.

By the open mapping theorem, we only have to prove that the map

$$
\Phi: X \widehat{\otimes}_{\mathcal{e}} \mathcal{H}(G) \rightarrow \mathcal{H}(G, X)
$$

is onto.

Let $f \in \mathcal{H}(G, X)$. We take a sequence $f_{n} \in X \otimes \mathcal{H}(G)$ so that

$$
\Gamma\left[\left(f-f_{n}\right)\left(K_{n}\right)\right] \subset B_{X}\left(0,2^{-n / p}\right) .
$$

From this, it follows that, if $n<m$,

$$
\Gamma\left[\left(f_{n}-f_{m}\right)\left(K_{n}\right)\right] \subset B_{X}\left(0,2^{-(n+1) / p}\right) .
$$


Now, if $\omega \in \Gamma \Delta_{n}$, we have that

$$
\left(I_{X} \otimes \omega\right)\left(f_{n}-f_{m}\right) \in \Gamma\left(f_{n}-f_{m}\right)\left(K_{n}\right) \subset B_{X}\left(0,2^{-(n+1) / p}\right) .
$$

If $\omega \in U_{n}^{0}$, we approximate it by $\omega_{\alpha} \in \Gamma \Delta_{n}$ in the weak topology and we obtain

$$
\left\|\left(I_{X} \otimes \omega\right)\left(f_{n}-f_{m}\right)\right\| \leqslant 2^{-(n+1) / p} .
$$

So, if $n<m$, then

$$
q_{n}\left(f_{n}-f_{m}\right) \leqslant 2^{-(n+1) / p}
$$

and $\left\{f_{n}\right\}_{n}$ is a Cauchy sequence in $X \otimes_{e} \mathcal{H}(G)$. Let $f_{n} \rightarrow f^{*}$ in $X \widehat{\otimes}_{e} \mathcal{H}(G)$ and $f_{n}=\Phi\left(f_{n}\right) \rightarrow \Phi\left(f^{*}\right)$ and $\mathcal{H}(G, X)$. Since $f_{n} \rightarrow f$ in $\mathcal{H}(G, X)$, the result is proved.

Finally, we recall that $\mathcal{H}(G)$ is a nuclear space and, by a result of Waelbroeck [16], the $p$-projective and the inductive topologies on $X \otimes \mathcal{H}(G)$ coincide.

Thus, we have the following result:

THEOREM 5.1. The spaces $X \widehat{\otimes}_{p} \mathcal{H}(G), X \widehat{\otimes}_{e} \mathcal{H}(G)$ and $\mathcal{H}(G, X)$ are topologically isomorphic. Any analytic function is globally holomorphic, and the uniform convergence on compact sets, the ultra-uniform convergence on compact sets, and the convergence with respect to the functionals $\dot{q}_{n}$ and $|\cdot| \widehat{\otimes}_{p}\|\cdot\|_{n}$, coincide, where

$$
\|\phi\|_{n}=\sup _{z \in K_{n}}|\phi(z)|, \quad \phi \in \mathcal{H}(G) .
$$

We remark that in [7] there is a proof of Theorem 5.1 that does not use nuclearity but only the approximation property of $\mathcal{H}(G)$. The inclusion

$$
X \widehat{\otimes}_{p} \mathcal{H}(G) \hookrightarrow \mathcal{H}(G, X)
$$

is a consequence of [7, Lemma 2.1] and Proposition 5.1. To obtain the equality, an argument involving projective limits is used.

\section{REFERENCES}

[1] A.B. Aleksandrov, 'Essays on non locally convex Hardy classes', in Complex Analysis and Spectral Theory: Lecture Notes in Math 864, Editors V.P. Havin and N.K. Nikolskii (Springer-Verlag, Berlin, Heidelberg, New York, 1981).

[2] A. Bernal and J. Cerdà, 'On non locally convex tensor products', (1989) (preprint).

[3] R.R. Coifman and R. Rochberg, 'Representation theorems for holomorfic and harmonic functions in $L^{p}$, Astérisque 77 (1980), 11-66.

[4] D.O. Etter, 'Vector-valued analytic functions', Trans. Amer. Math. Soc. 119 (1965), 352-366. 
[5] B. Gramsch, 'Integration und holomorphe funktionen in lokalbeschränkten Räumen', Math. Annal. 162 (1965), 190-210.

[6] B. Gramsch, 'Tensorprodukte und integration vektorwertiger funktionen', Math. Z. 100 (1967), 106-122.

[7] B. Gramsch and D. Vogt, 'Holomorphe funktionen mit Werten in nicht lokalkonvexen Vektorräumen', J. Reine Angew. Math. 243 (1970), 159-170.

[8] N.J. Kalton, 'Analytic functions in non-locally convex spaces and applications', Studia Math. 83 (1986), 275-303.

[9] N.J. Kalton, 'Plurisubharmonic functions on quasi-Banach spaces', Studia Math. 84 (1986), 297-324.

[10] N.J. Kalton, N.T. Peck and W. Roberts, 'An F-space sampler', London Math. Soc. Lecture Note Ser. 89. (Cambridge Univ. Press 1984).

[11] J. Peetre, 'Locally analy tically pseudo-convex topological vector spaces', Studia Math. 73 (1982), 252-262.

[12] P. Turpin, 'Convexités dans les espaces vectoriels topologiques généraux', Dissertationes Math. (Rozprauy Mat.) 131 (1974).

[13] P. Turpin, 'Produits tensoriels d'espaces vectoriels topologiques', Bull. Soc. Math. France 110 (1982), 3-13.

[14] P. Turpin, 'Représentation fonctionnelle des espaces vectoriels topologiques', Studia Math. 73 (1982), 1-10.

[15] D. Vogt, 'Integrationstheorie in p-normieten Räumen', Math. Ann. 173 (1967), 219-232.

[16] L. Waelbroeck, 'The tensor product of a locally pseudo-convex and a nuclear space', Studia Math. 38 (1970), 101-104.

Department de Matemàtica Aplicada i Anàlisi

Universitat de Barcelona

08071 Barcelona

Spain 